\title{
Editorial: The Role of Colloquia in Reviews of Modern Physics
}

We all have had this experience: we walk out of a colloquium talk that covered a topic far from our own field and say to the colleague who happens to be next to us, "This was a great talk. The speaker really managed to convey why this stuff is interesting. I can see why the people who are working on it are excited about it." This does not happen after every talk, but it does happen regularly.

RMP Colloquia are intended to be written versions of such exceptional colloquium talks. What sets them apart from the R in RMP, i.e., full-length reviews, is that they are not intended to give a comprehensive account of a topic. Rather, they are meant to be exemplary, kindle the interest of a broad audience, and shine a spotlight on aspects of a topic that the authors find particularly fascinating and important. They were the brainchild of Ugo Fano, who served as an associate editor for RMP from 1990 to 1995, when David Pines was Lead Editor. Since their inception in 1992 they have been an important part of RMP, overseen by three colloquium editors following Fano: Anthony Starace (1996-2006), Bruce Shore (2006-2010), and Antonio Castro Neto (2010-2020). Colloquia are still overseen by a dedicated colloquium editor, but the management structure has recently changed to provide more involvement of the topical associate editors. Authors interested in proposing a colloquium article should feel free to contact either the colloquium editor, who will coordinate with the appropriate topical editor(s), or vice versa.

We would like to take the opportunity to thank all previous colloquium editors, whose work has made the colloquia section as vibrant as it is. We are looking forward to maintaining and further developing this important part of our journal.

\author{
Dietrich Belitz \\ Colloquium Editor \\ Randall D. Kamien \\ Lead Editor
}

Published 4 January 2021

DOI: 10.1103/RevModPhys.93.010001 